\title{
Qualitative analysis of Fruits and Vegetables using Earth's Field Nuclear Magnetic Resonance (EFNMR) and Magnetic Resonance Imaging (MRI)
}

\author{
L. P. Deshmukh, K. R. Bagree and S. J. Sharma \\ Department of Electronics and Computer Science, Rashtrasant Tukadoji Maharaj Nagpur University, Nagpur- \\ 440033 (India)
}

\begin{abstract}
Among the imaging techniques, magnetic resonance imaging (MRI) is a non-contact and a non-invasive technique to obtain images of the objects rich in water content and provides an excellent tool to study variation of contrast among the soft issues. It often utilizes a linear magnetic field gradient to obtain an image that combines the visualization of molecular structure and dynamics. It measures the characteristics of hydrogen nuclei of water and nuclei with similar chemical shifts, modified by chemical environment across the object. In the present work, MRI of fresh tomatoes has been recorded using Terranova-MRI for qualitative analysis. The technique is effective, powerful and reliable as an investigative tool in the quality analysis and diagnosis of infections in fruits and vegetables.
\end{abstract}

Keywords: Non-invasive imaging, Earth's Field Nuclear Magnetic Resonance (EFNMR), Magnetic Resonance Imaging (MRI), qualitative analysis, tomatoes, tomographic slice, Terranova- MRI, Prospa

\section{INTRODUCTION}

There are various imaging techniques in biomedical field such as ultrasonic [1-5], X-rays [611] and NMR [12-21]. Among the imaging techniques, magnetic resonance imaging (MRI) is effective, powerful and reliable. Biomedical engineers find better opportunities in the design of this imaging technique. MRI is a non-invasive imaging technique most commonly used in biomedical research and medical radiology to visualize structures and functions of the living system and single cells. It is also used to produce tomographic images from human or other biological systems rich in water content. MRI provides an excellent contrast among the various soft tissues as compared to the computer tomography (CT) where images are used to ascertain the difference in tissue density to reflect X-rays.

MRI machines make use of the fact that body tissues contains lot of water (and hence protons) which gets aligned in a large magnetic field. Each water molecule has two hydrogen nuclei or protons and when a person is inside the powerful magnetic field of the scanner, the average magnetic moment of many protons is aligned with the direction of the applied magnetic field. A radio frequency current is briefly turned on, producing a varying electromagnetic field. This electromagnetic field has just the right frequency known as resonance frequency, to be absorbed and flip the spin of the protons in applied the magnetic field.
After the electromagnetic field is turned off, the spin of the protons returns to the thermodynamic equilibrium and the bulk magnetization becomes realigned with a static magnetic field. During the relaxation, a radio frequency signal is generated which can be measured with the help of receiver coils. Information about the origin of the signal in 3D space can be obtained by applying additional magnetic fields during the scan. 3D image can also produce images in any plane of view. This image can be rotated and manipulated to detect the tiny changes of structures within the body.

In the present work, MRI images of the fresh tomatoes are recorded using Terranova-MRI manufactured by Magritek, New Zealand.

\section{EXPERIMENTAL:}

Terranova-MRI is basically the Earth's Field Nuclear Magnetic Resonance (EFNMR) [2224] and NMR measuring system. It consists of three main hardware components: A coil probe, a digital signal processing (DSP) unit and a Personal Computer. The coil probe consists of three sets of coils that slot together in a bayonet configuration. The outermost coil is coaxial solenoid, called polarizing coil. If applied with a large current, polarizing coil will produce an initial polarizing field to enhance the net nuclear magnetization of the subject sample in the probe. Middle coils are the gradient coils to generate four magnetic field gradients. The $\mathrm{X}, \mathrm{Y}$ and $\mathrm{Z}$ gradients are applied around the sample for encoding of the spatial 
information, the fourth gradient coil is for shimming. The inner coil is another coaxial solenoid, called B1. It generates the pulse that excites and detects the magnetic resonance signals. The digital signal processor (DSP) is the central control unit between the coil probe and the computer. It receives precompiled pulses, programs and parameters from computer. After processing these programs, DSP unit sends related pulses to the coils of the probe that acquire the magnetic resonance signals. Most of the operations of the MRI system are accomplished using GUI control panel developed in data processing package, Prospa.

Obtaining the first free-induction decay (FID) and spectrum is the most challenging step in the set-up process for the EFNMR system. Many inter-dependent parameters and factors contribute to the quality of the acquired EFNMR signal.

- Coarse tuning of the probe:

The first step once the system is switched on is to tune B1 coil at the observed resonant frequency, to obtain maximum signal. The tuning macro can be found in the EFNMR menu under the name Analyze Coil.

This experiment applies an impulse to the B1 coil and gives the response as a function of the tuning capacitance (which is under computer control). The output of the Analyze Coil is as shown below.
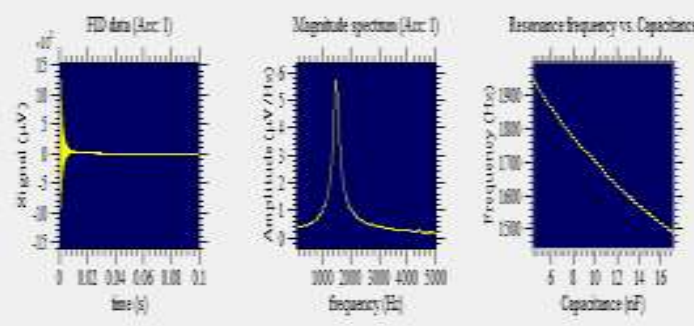

Fig 1: Sampled output from the Analyze Coil

The three sub-plots in Fig. 1 represent the time domain response to the impulse, the frequency domain response, and the peak of the response versus tuning capacitance curve. From this curve the program calculates the inductance and parasitic capacitance of the B1 coil.

- Selection of an initial location for the EFNMR system:

The EFNMR instrument is very sensitive to the location. There are two location dependent factors which greatly affect the quality of the observed signal: local magnetic field inhomogeneity and noise caused by interference from electronic equipment in the vicinity of the EFNMR probe.
A procedure called Monitor Noise in the EFNMR menu is performed to determine the best location.

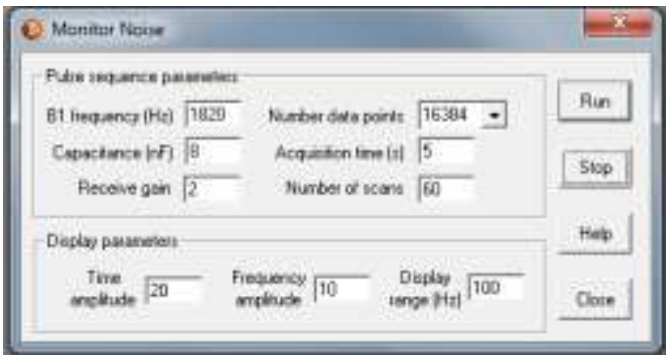

Fig. 2 (a) : The Monitor Noise macro

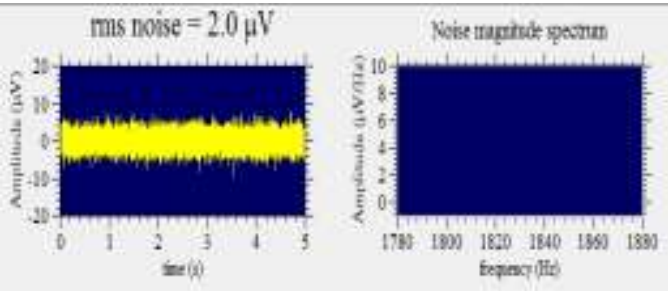

Fig. 2 (b): Output from the Monitor Noise experiment

A typically acceptable noise level for MRI is less than $10 \mu \mathrm{Vrms}$, anything less than $5 \mu \mathrm{V}$ is good and less than $3 \mu \mathrm{V}$ is excellent. Noise levels between 10-100 $\mu \mathrm{V}$ will make basic NMR measurement difficult and MRI almost impossible.

- Select initial parameters:

Initially, insert a standard bottle filled with tap water into the probe. The supplied bottle with 73 $\mathrm{mm}$ diameter, $570 \mathrm{ml}$ volume bottle is an ideal choice of a sample container. The pulse and collect steps are the simplest NMR pulse sequence, consisting of polarizing pulse and an excitation, or B1, pulse followed by the detection of the signal.

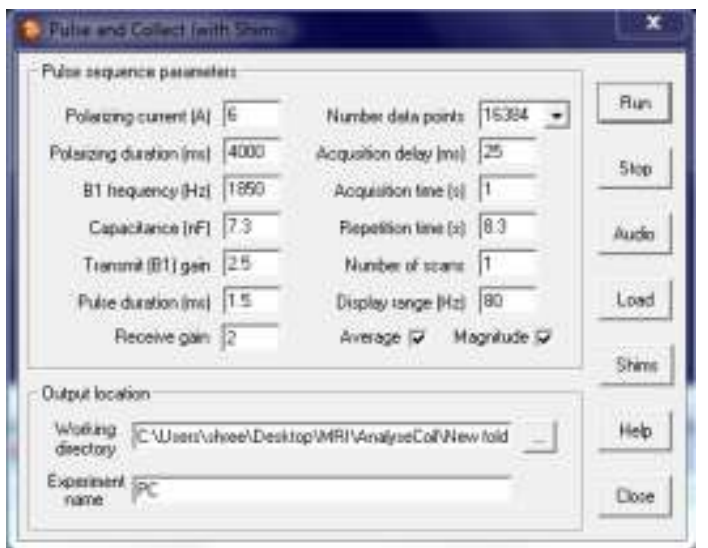

Fig. 3: The pulse and collect macro

The polarization controls the duration and the strength of an initial polarizing pulse in an EFNMR experiment. For tap-water, the duration of 
the polarizing pulse is set to $4000 \mathrm{mS}$ in order to maximize the signal intensity and enhancement. The polarizing current parameter takes values from 0 to 6.0 A. The B1 frequency should be set to the Larmor frequency of the sample. The Larmor frequency is dependent on the gyro magnetic ratio of the observed nucleus and the strength of the Earth's magnetic field. Typically this value will be in the range 1800 to $2500 \mathrm{~Hz}$.
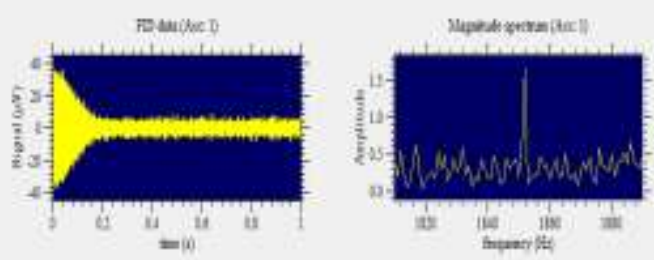

Fig. 4 (a): The output of Pulse and collect procedure

As mentioned earlier, it is however very likely that no signal is observed or that the observed signal is very small. The problem is likely due to be local magnetic field in-homogeneity.
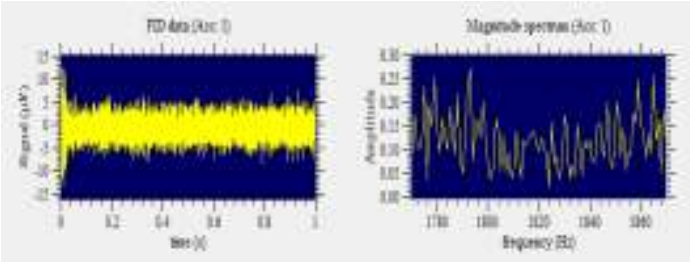

Fig. 4 (b): The output of Pulse and collect steps in the region of magnetic field in-homogeneity

- Using a spin-echo to find a signal

It is difficult to optimize the location and other experimental parameters of the EFNMR system without an observable signal. Therefore, in order to effectively troubleshoot the setup of the instrument, it is useful to employ the other NMR method called as "spin-echo" experiment.

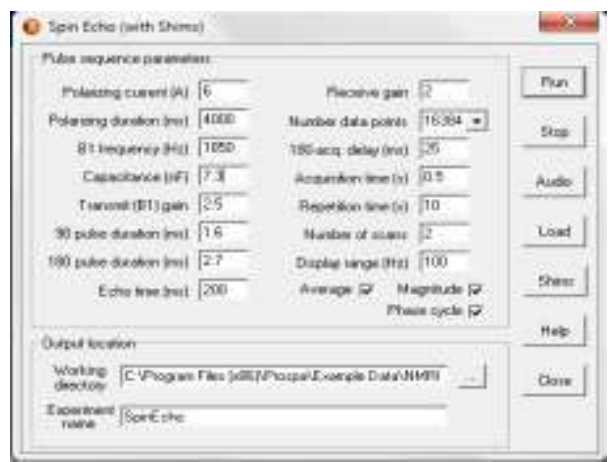

Fig. 5(a): The spin-echo macro

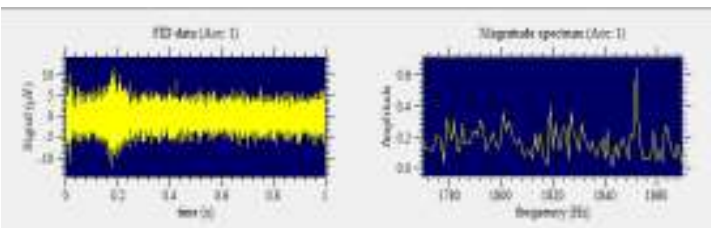

Fig. 5 (b): The result of spin-echo procedure

- Shimming:

This macro uses an iterative procedure to modify the current through the $\mathrm{x}, \mathrm{y}$ and $\mathrm{z}$ gradients until the peak height in the frequency domain is maximized.

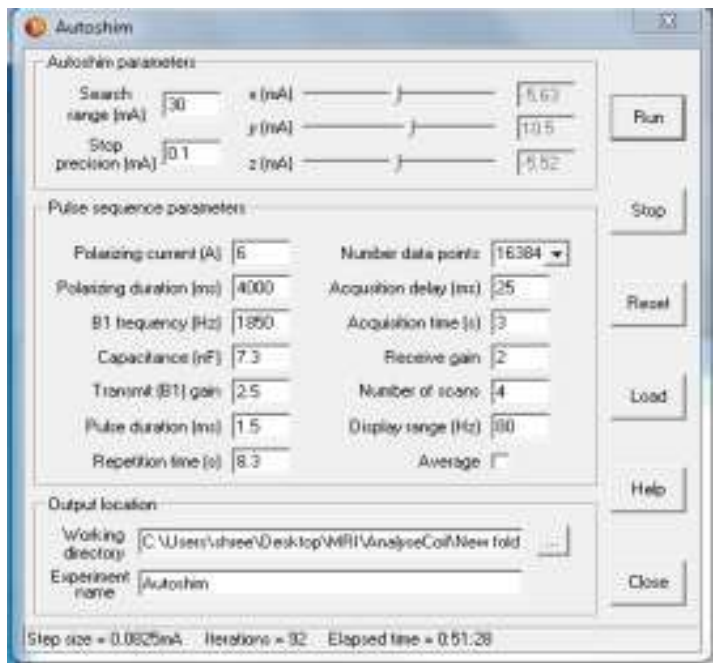

Fig. 6 (a): The Auto shim macro

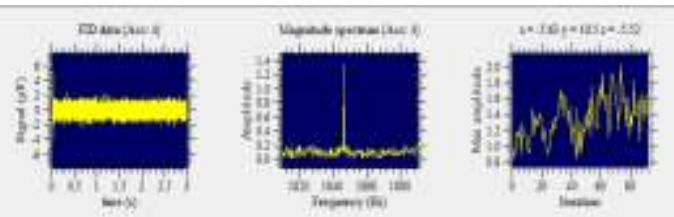

Fig. 6 (b): The output from the Auto shim process

- B1 Duration:

Using this macro, the signal amplitude is determined by integrating the spectral peak over a range of frequency values. The B1 parameters define the array of the $\mathrm{B} 1$ pulse durations to be used for the experiment.

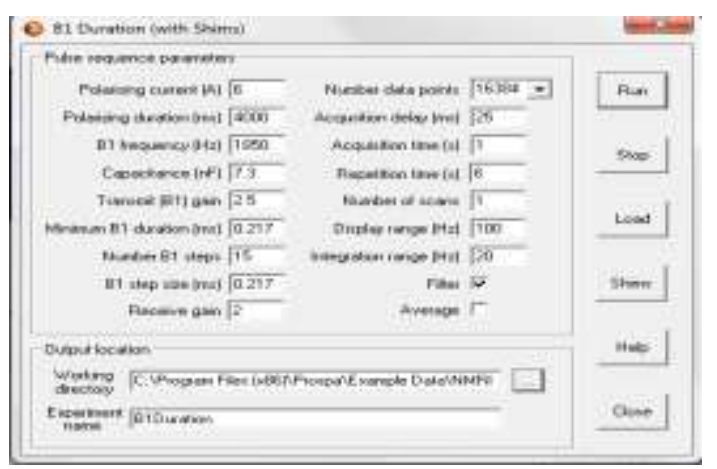

Fig. 7(a): The B1 Duration macro 

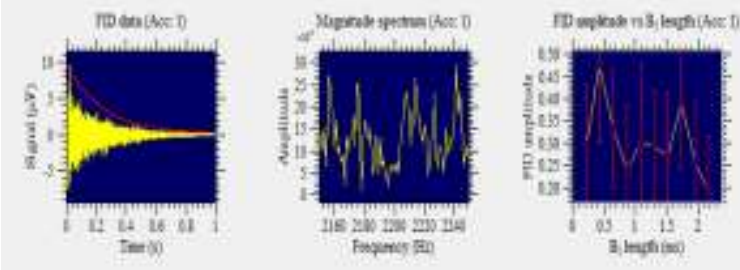

Fig. 7 (b): The output from the B1 duration experiment

\section{CONCLUSION}

The facility of MRI of the instrument was tested in the laboratory on freshly obtained tomatoes from the local market. Fig. 8 shows the recording of MRI of the fresh tomato under study using Terranova-MRI instrument, Magritek, from New Zealand. The image looks slightly blurred but one can clearly see the tomographic slice along with the dimensions of the tomato under study. The quality of the image can be improved if the image processing software, Pospa, is incorporated with additional features to refine the image.
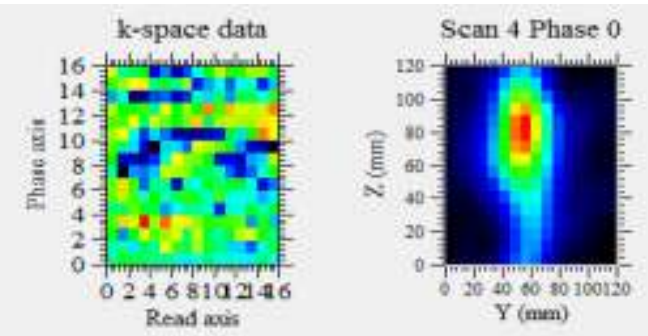

Fig. 8: $2 D \mathrm{YZ}$ tomato image (4 scans)-12 minutes

As the EFNMR system is working on the Earth's magnetic field, the pulse frequency is low and the nuclear magnetization is weak. However, there are many difficulties associated with obtaining and analyzing high-quality NMR spectra in such a weak field. The challenge associated with EFNMR is the optimization of resolution and sensitivity. While the Earth's field itself is highly spatially homogeneous, this homogeneity is easily disrupted by magnetic objects. Additionally, very low Larmor frequencies of nuclei in the Earth's field render EFNMR measurements highly susceptible to interference from external ULF noise sources. The electric noises from the surrounding environment are high enough to disturb the magnetic resonance signal of this system. The instrument is very useful in the qualitative analysis and study of MRI of fruits and vegetables in a laboratory. This facility of the instrument proves to be effective and useful for the diagnosis and study of growth of pathogens and degree of infections in fruits and vegetables.

\section{REFERENCES}

[1]. Nigam A. K., Ultrasonic Imaging System, US Patent 4, 1978, 0482, 582

[2]. Engeler W. E. Ultrasonic Imaging System, US Patent 4, 1979, 155, 259

[3]. Smith S. W., Pavy H. G. and von Ramm O. T., High Speed Ultrasound Volumetric Imaging System I. Transducer Design and Beam Steering, IEEE Trans. Ultraso. Ferro. \& Freq. Contr. 38(2), 1991, 100-108

[4]. Von Ramm O. T., Smith S. W. and Pavy H. G., High Speed Ultrasound Volumetric Imaging System II. Parallel Processing \& Image Display, IEEE Trans. Ultraso. Ferro. \& Freq. Contr. 38(2), 1991, 109-115

[5]. Reinholdtsen P. A. and Khuri-Yakub B. T., Image processing for a Scanning Acoustic Microscope that Measures Amplitude \& Phase, IEEE Trans. Ultraso. Ferro. \& Freq. Contr. 38(2), 1991, 141-147

[6]. Zhao J., Opsahl-Ong B. H. and Hopple M. R., X-ray Imaging System and Method for Radiography and Mammography, US Patent 09/586, 898, 2000

[7]. Miller E. A., White T. A., McDonald B. S. and Seifert A., Phase Construct X-ray Imaging Signatures for Security Applications, IEEE Trans. Nucl. Sci. 60, 2013, 416-422

[8]. Fu J. and Wang J. Z., Accurate Image Construction of few-view Ptychrography X-ray Computed Tomography, IEEE Intl. Conf. Info. \& Automation (ICIA), 1505 1509, 2016

[9]. Gjesteby L., Xi Y., Kalra M. K., Yang Q. and Wang G., Hybrid Imaging System for Simultaneous Spiral MR \& X-ray (MRX) Scans, IEEE Access, 5, 2017, 1050-1061

[10]. Mohammadi H.M. and de Guise J. A., Enhanced X-ray Image Segmentation Method using Prior Shape, IET Comp. Vision, 11(2), 2017, 145-152

[11]. Hashem J. A., Pryor M., Landsberger S., Hunter J. and Janecky D. R., Automatic High-Precision X-ray \& Neutron Imaging Application with Robotics, IEEE Trans. On Automation Sci. \& Engg. 99, 2017, 1-12

[12]. Geva, T., Magnetic Resonance Imaging: Historical perspective, J. of Cardv. Magn. Reso., 8(4), 2006, 573-580

[13]. Roguin, A., Nikola Tesla: The Man Behind the Magnetic Field Unit, J. Magn. Reso. Imag., 19(3), 2004, 369-374

[14]. Nie. Zhuang, MRI Simulation by the EFNMR system and MATLAB for Medical 
Imaging Teaching (2012) Open Access theses

[15]. Liang, Z. P. and P. C. Lauterbur, IEEE Engineering in Medicine and Biology Society., Principles of Magnetic Resonance Imaging: A Signal Processing Perspective, IEEE Press Series in Biomedical Engineering 2000, Bellingham, Washington, USA

[16]. Liao S. H, Chen M. J, Yang C. H., A Study of J-coupling Spectroscopy using the Earth's Field Nuclear Magnetic Resonance inside a Laboratory, Rev. Sci. Instrum. 81, 2010, 104104

[17]. Gossuin, Y., Hocq A., Gillis P. And Vuong Q. L., Physics of Magnetic Resonance Imaging: From Spin to Pixel, J. Phys. DAppl. Phys., 43(21),2010, 213001

[18]. Hinshaw, W. S. and Lent A. H., An Introduction to NMR imaging: From the Bloch Equation to the Imaging Equation, Proc. IEEE, 71(3), 1983, 338-350

[19]. Williams, J. E. C., Superconducting Magnets for MRI, IEEE Trans. Nucl. Sci., 31(4), 1984, 994-1005

[20]. Hidalgo-Tobon S. S., Theory of Gradient Coil Design Methods for Magnetic Resonance Imaging. Concepts, Magn. Reso., 36A, 2010, 223-242

[21]. Rutt B. K. and Lee D. H., The Impact of Field Strength on Image Quality in MRI, $J$. Magn. Reso. Imag.,6, 1996, 57

[22]. Terranova-MRI, Earth's Field MRI \& NMR teaching system, User manual

[23]. Terranova-MRI, Earth's Field MRI \& NMR teaching system, Student guide

[24]. Terranova-MRI, Earth's Field MRI \& NMR teaching system, Prospa 